\title{
Angioinvasive Mold in the Surgical and Burn Intensive Care Unit: A Case Series and Review of the Literature
}

\author{
Jason C. Gardenier,', Vinod K. Chopra,' Filippo Filicori,, Jennifer Murphy, Andrew Greenway, \\ James J. Gallagher, ${ }^{1}$ Palmer Q. Bessey, Abraham Houng, Soumitra R. Eachempati, ${ }^{1}$ and Philip S. Barie ${ }^{2}$
}

\begin{abstract}
Background: Angioinvasive mold infections (AMIs) are among the most serious infections a patient can acquire. These infections carry high mortality and are difficult to cure.

Methods: Report of case series of five patients with AMIs and review of pertinent English-language literature. Results: Five cases of AMIs developed in the surgical and burn intensive care units (ICUs) of a single center over a three-year period; four patients were female. The mean age was $48 \mathrm{y}$. One patient had advanced breast cancer and four had large total body surface area (TBSA; average 59\%) burns. Among the burn patients, AMIs developed on average $25 \mathrm{~d}$ after burn injury (range, 18-35d). Four of the AMIs reported here were polymicrobial; four patients had Candida spp., three had Aspergillus spp., two had Fusarium spp., two had Trichosporon asahii, two had Mucor spp., and one had Purpureocillium lilacinus. Four patients were treated with intravenous voriconazole, two with intravenous amphotericin B (amB), and one was treated with enteral posaconazole. Adjunctive topical anti-fungal therapy consisted of silver nitrate solution $(n=4), 0.5 \%$ sodium hypochlorite (Dakin) solution $(n=3)$, and topical $\operatorname{amB}(n=1)$.

Conclusion: Three patients were cured of their infections (one of whom succumbed to a bacterial infection later in the hospitalization) and two died of the AMI, for an overall mortality rate of $60 \%$. A majority of AMIs were polymicrobial, which has not been reported previously. To our knowledge, this is the first case report of $P$. lilacinus AMI in a burn patient.
\end{abstract}

A NGIOINVASIVE MOLD INFECTIONS (AMIs) are rare but aggressive infections that carry a high mortality rate (76\%-88\% in some series) [1-3]. They usually affect immunosuppressed patients, including patients with poorly controlled diabetes mellitus, transplant recipients, and patients with cancer, but can also affect severely injured, circumstantially immunosuppressive patients after trauma [4] and burn injuries of large total body surface area (TBSA) $[5,6]$. Angioinvasive mold infections appear frequently after commensal bacterial flora have been obliterated with broad spectrum antibiotics [2]. Aspergillus spp., Fusarium spp., and zygomycetes are the most common pathogens, which have differing susceptibilities to anti-fungal agents. Vascular invasion may cause widespread dissemination and systemic coagulation disorders as well as thrombosis, promoting tissue necrosis [7].

In this report, we discuss a three-year, single-center experience of five patients with AMI. Diagnosis was made by biopsy with hyphal elements identified with potassium hydroxide $(\mathrm{KOH})$ preparation (Fig. 1A); speciation and antimicrobial susceptibilities were obtained from the clinical microbiology laboratory of our facility (Fig. 1B). The presence of invasive mold was confirmed with hematoxylin and eosin (Fig. 1C), periodic acid-Schiff (PAS; Fig. 1D), or Golcott-Gomori

${ }^{1}$ Department of Surgery, Division of Burns, Trauma, Acute and Critical Care, ${ }^{2}$ Department of Medicine, Division of Medical Ethics, Weill Cornell Medicine, New York-Presbyterian Hospital/Weill Cornell Medical Center, New York, New York.

(c) Jason C. Gardenier et al. 2016; Published by Mary Ann Liebert, Inc. This Open Access article is distributed under the terms of the Creative Commons License (http://creativecommons.org/licenses/by/4.0), which permits unrestricted use, distribution, and reproduction in any medium, provided the original work is properly credited. 

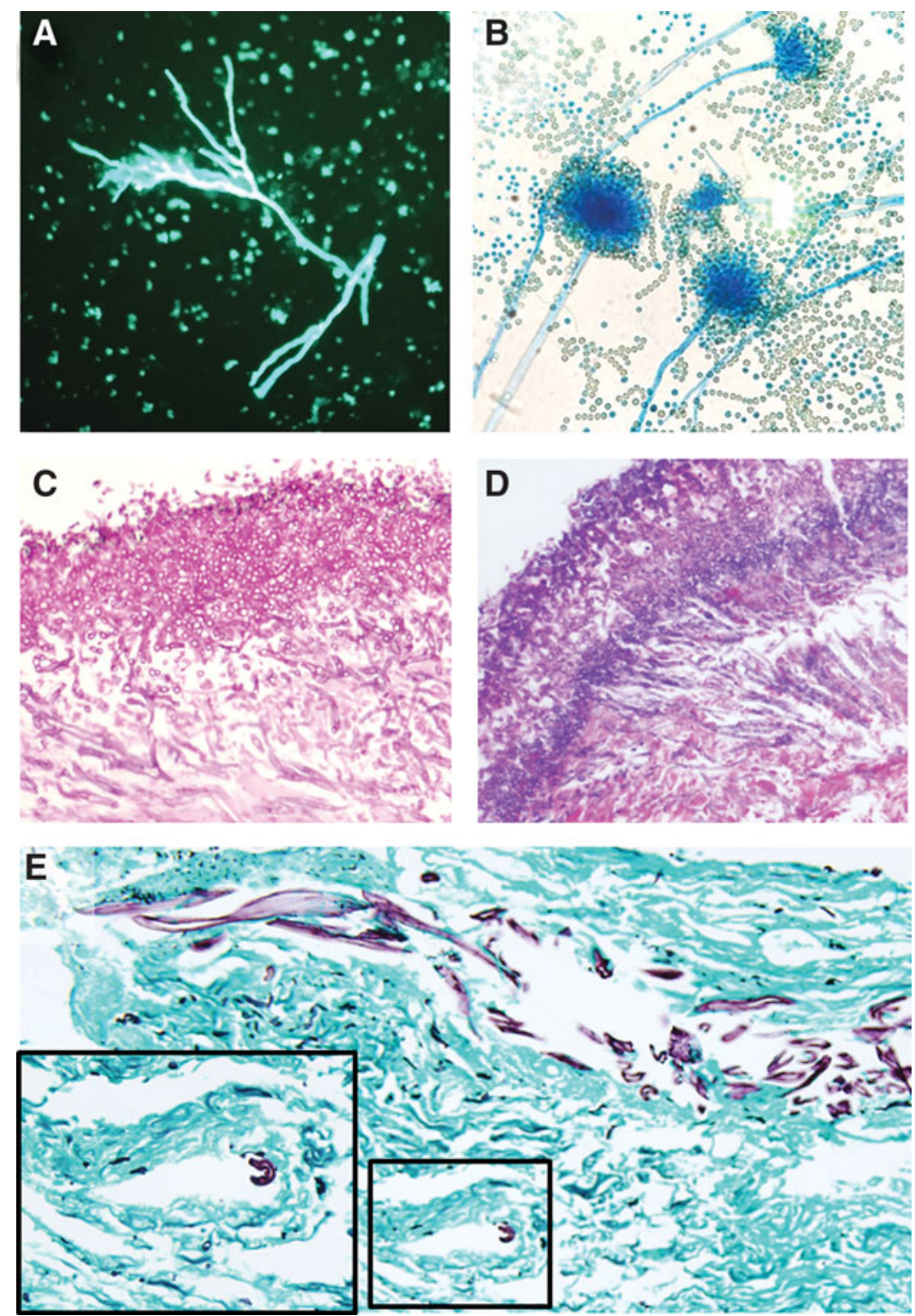

FIG. 1. (A) Potassium chloride $(\mathrm{KOH})$ preparation showing fungal hyphae with branching (original magnification: $400 \times$ ). (B) Aspergillus calodostus conidia growing in culture (original magnification: 400x). (C) Hematoxylin and eosin staining showing dense fungal hyphae with invasion of the underlying tissue (original magnification: 400×). (D) Periodic acid-Schiff staining of a tissue biopsy showing dense fungal elements (purple) invading underlying soft tissue (pink, original magnification: 200×). E) Grocott-Gomori methenamine silver (GMS) staining with a methyl green counterstain of a soft tissue biopsy from a patient with angioinvasive mold demonstrating black hyphae (original view: 400x). Inset photo: A greater magnification of a fungal element within a small-caliber blood vessel.

methenamine silver (GMS, Fig. 1E) stains. Patient characteristics, treatments, and outcomes are discussed.

\section{Case 1}

A 70-year-old obese Caucasian female with a history of right breast cancer presented with a necrotizing soft tissue infection (NSTI) of a sacral decubitus ulcer. After a recent fall at home and in an attempt to avoid additional falls, the patient decided to remain seated on the floor. Her husband visited her several times but did not call for medical assistance; rather, he elected to leave food for her and intermittently to cleanse her of urine and stool while leaving her seated on the floor for more than two weeks. The sacrum and both buttocks developed pressure sores, which were soiled repeatedly and a NSTI developed. After sepsis overwhelmed the patient, she presented by ambulance for medical treatment. A malignant right breast neoplasm had been treated in the past by local excision alone, with apparent recurrence some time previously without medical treatment. The patient thus also had a large fungating right breast mass and a large right-sided malignant pleural effusion, which led to atelectasis.

On hospital day one the patient was intubated, received fluid resuscitation, had a right tube thoracostomy, was pan-cultured, started on broad spectrum antibiotics and vasopressors, and went to the operating room for surgical debridement of her 

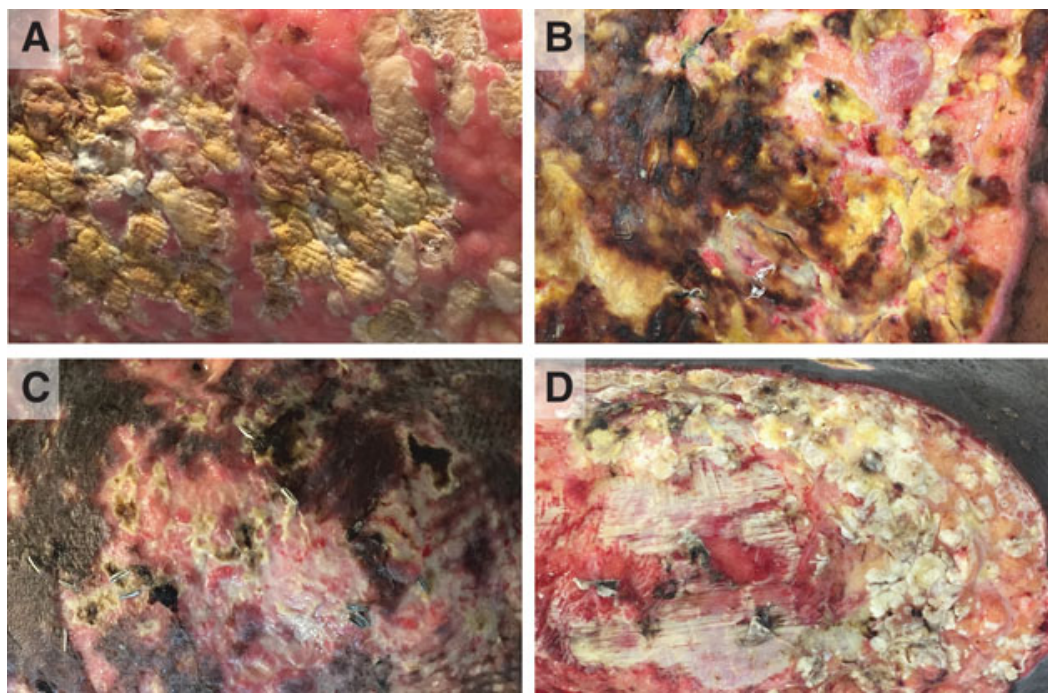

FIG. 2. Representative photographs of the mold infections infecting patients detailed in cases 2-5 (A-D, respectively).

bilateral buttocks, perineum, and right thigh. Blood cultures yielded Proteus mirabilis, and wound culture revealed polymicrobial flora including Escherichia coli, P. mirabilis, vancomycin-susceptible Enterococcus faecalis, and Bacteroides fragilis. She was treated with clindamycin, daptomycin, piperacillin-tazobactam, and micafungin, then de-escalated to piperacillin-tazobactam monotherapy based on initial microbiology data. The surgical site was dressed with diluted $(0.025 \%)$ sodium hypochlorite (half-strength Dakin) solution. Serial debridements were performed over the next two weeks. A tissue culture taken on hospital day 12 grew many Mucor spp., Candida glabrata, and C. parapsilosis, at which time she was started on intravenous amphotericin B $(\mathrm{amB})$ and enteral posaconazole. Because of persistent visible mold colonies, topical treatment was begun with an amB from hospital day 20 until hospital day 44 . She returned to the operating room on hospital day 22 for tracheostomy and on hospital day 29 underwent gastrostomy and sigmoid end colostomy for persistent surgical site contamination and sepsis. She recovered and returned to the operating room on hospital day 43 for splitthickness skin grafting of her perineum and leg wounds.

As it appeared she would survive, on hospital day 56 she underwent a palliative right total mastectomy. Septic shock developed as a result of polymicrobial multi-drug-resistant Pseudomonas aeruginosa pneumonia on hospital day 81 and acute kidney injury developed as a result of her vasopressor requirement, however, treatment with polymyxin B and tigecycline was successful. After recovery and discontinuation of renal replacement therapy, the patient was discharged to a sub-acute rehabilitation center on hospital day 103.

\section{Case 2}

A 35-year-old South Asian female with no prior medical history suffered 93\% TBSA full-thickness flame injuries from self-immolation. Only the scalp, breasts, perineum, glabrous skin of hands and feet, and a narrow strip on the dorsal left forearm were not burned. She was alert and responsive, claimed her injuries were accidental, and requested aggressive care, which was endorsed by her family. She was intubated and received a $5.7 \mathrm{~mL} / \mathrm{kg} / \%$ burn resuscitation in the first $24 \mathrm{~h}$. She required fasciotomies of her right lower extremity for compartment syndrome in addition to standard escharotomies of the chest, abdomen, and all four extremities. Although there was no inhalation injury, she required high-pressure bilevel ventilation for $10 \mathrm{~d}$. She underwent excisions on post-burn days two, four, and nine. At each excision, cadaveric split-thickness skin grafts (i.e., homograft) were applied to all sites. On post-burn day 11, autografting was attempted on her anterior trunk, harvesting all available unburned skin and performing hand micrografting. Similar autografts were performed on post-burn day 18 (with a tracheostomy), and post-burn day 25. Despite autografitng, the patient suffered recurrent bouts of septic shock caused by several unusual opportunistic organisms (see below), with resultant failure of the autografts to grow sufficiently to coalesce and reconstitute an effective epidermal barrier over the majority of the body's surface.

The first documented infection was on post-burn day 7 , when cultures revealed Enterobacter aerogenes bacteremia (treated with intravenous ceftazidime and tobramycin based on susceptibilities), $C$. parapsilosis fungemia (treated with intravenous fluconazole based on susceptibility), and Acinetobacter baumanii pneumonia (also treated with ceftazidime and tobramycin). The patient developed Proteus fluorescens bacteremia on post-burn day 9 (ceftazidime was switched to cefepime), and a Strenotrophomonas maltophilia bacteremia (treated with intravenous trimethoprim-sulfamethoxazole) on post-burn day 10 . Thereafter she developed a susceptible $P$. aeruginosa pneumonia on post-burn day 12 . Although the initial bacteremia/fungemia cleared, she developed bacteremic pneumonia with Elizabethkingia meningoseptica on post-burn day 20 (treated with intravenous amikacin, then switched to piperacillin-tazobactam plus minocycline based on susceptibilities) and a vancomycin-intermediate Enterococcus gallinarum (treated with intravenous daptomycin). The second bacteremia also cleared with negative blood cultures from post-burn day 32 and 34 .

A surface lesion suspicious for mold was biopsied on postburn day 35; cultures eventually grew $C$. guilliermondii, Aspergillus flavus, and Aspergillus calidoustus. Pathology confirmed an AMI. Topical silver nitrate and intravenous 
voriconazole were administered. The AMI was debrided to fascia on post-burn day 37, but on post-burn day 40 additional lesions confirmed to be AMI were noted on the feet, ankles, and knees and on $\mathrm{KOH}$ preparation, fungal components were observed on her bilateral ankles and knees. Because the mold was growing on fascia and joint capsules of the lower extremities, soft tissue debridement was determined not to have curative potential, so bilateral above-knee amputations were performed on post-burn day 41, eradicating the AMI.

However, the patient continued to suffer repeated infections, including Clostridium difficile colitis (post-burn day 39), Stenotrophomonas maltophilia pneumonia (post-burn day 48), and vancomycin-resistant Enterococcus faecalis bacteremia (post-burn day 58). The $C$. difficile infection was unresponsive to all therapies; refractory hypothermia required placement of a central venous thermoregulation catheter (Thermogard XP ${ }^{\circledR}$ Temperature Managament System, Zoll Medical Corp, Chelmsford, MA). On post-burn day 71 she developed a recurrent E. meningoseptica bacteremia and on post-burn day 77 was found to have recurrent $S$. maltophilia bacteremic pneumonia. At this point, no escalation of care was decided upon and the patient died on postburn day 85 .

\section{Case 3}

A 33-year-old African American female with no prior medical history sustained a 68\% TBSA flame burn after self-immolation. She received an $8.57 \mathrm{~mL} / \mathrm{kg} / \%$ burn resuscitation in the first $24 \mathrm{~h}$. She required intubation and escharotomies of her arms, hands, chest, abdomen and thighs; bilateral lower extremity four-compartment fasciotomies; and right forearm dorsal and volar compartment fasciotomies for extremity compartment syndrome; a decompressive laparotomy for abdominal compartment syndrome; bilateral lateral canthotomies for orbital compartment syndrome; bilateral chest tube thoracostomies for bilateral pleural effusions and hypoxemia. She developed rhabdomyolysis (peak serum creatine phosphokinase concentration, 20,567 IU/L) and developed acute kidney injury on post-burn day two, necessitating continuous renal replacement therapy (CRRT). Burn excisions were performed on post-burn days three, five, and eight with homograft placement each time. Profound refractory hypothermia required placement of a central venous thermoregulation catheter on post-burn day eight. The abdominal fascia was closed on post-burn day 10 and on postburn day 15 a tracheostomy was performed. She underwent autografting on post-burn days 17 and 22. However, recurrent infections led to marked clinical deterioration (see below).

Urine culture yielded $P$. aeruginosa on post-burn day 14 (treated with intravenous piperacillin-tazobactam). On postburn day 19 Klebsiella pneumoniae pneumonia was diagnosed (also treated with intravenous piperacillin-tazobactam). On post-burn day 22, intravenous fluconazole was started for the finding of yeast in broncheoalveolar lavage fluid and urine. On post-burn day 24 , a surface lesion concerning for AMI was biopsied on her left thigh and confirmed to be so. Topical dressings were switched to sodium hypochlorite and silver nitrate and the intravenous fluconazole was switched to intravenous voriconazole. Cultures yielded Fusarium spp. and $C$. albicans. Multiple AMI lesions were identified on bilateral lower extremities on post-burn day 27 . On post-burn day 28 she was found to have fungemia with Trichosporon asahii and C. albicans. Sepsis continued to worsen and care was withdrawn by her family. She died of septic shock on post-burn day 29 .

\section{Case 4}

A 65-year-old African American female with hypertension, atrial fibrillation treated with warfarin, and chronic obstructive pulmonary disease sustained a 20\% TBSA mostly full-thickness flame burn to the bilateral lower extremities and buttocks. She received a $3.1 \mathrm{~mL} / \mathrm{kg} / \%$ burn resuscitation in the first $24 \mathrm{~h}$ but did not require intubation or escharotomies. Excision of eschar and autografting were performed on post-burn days four and 11. On post-burn day 17 pneumonia was clinically diagnosed and was treated with piperacillin-tazobactam and vancomycin, although there was no yield from bronchalveolar lavage. On post-burn day 18 substantial graft loss was accompanied by areas on the right thigh suspicious for AMI. The topical antimicrobial regimen was switched to silver nitrate and the patient was started on intravenous voriconazole. Pathology showed invasive mold, and the tissue culture yielded A. flavus. Excision of the moldinfected soft tissue and placement of homograft was performed on post-burn day 24. Repeat autografting was performed on post-burn day 31. Thereafter, she again had partial graft loss and was monitored for an extended period of time but did not require re-operation. On post-burn day 41 warfarin was restarted and transfer to a sub-acute rehabilitation center occurred on post-burn day 50.

\section{Case 5}

A 38-y-old African American male with no prior medical history suffered 53\% TBSA flame burns to the bilateral upper and lower extremities, genitals, and back in a house fire after losing consciousness from inhalation injury. The majority of his burns were full thickness and his circumferential leg burns were particularly severe. He suffered cardiac arrest in the field and received cardiopulmonary resuscitation for $20 \mathrm{~min}$ before return of spontaneous circulation. He received hydroxocobalamin $5 \mathrm{~g}$ in the field $\left(\right.$ Cyanokit $^{\circledR}$, Meridian Medical Technologies, Columbia, MD) and arrived at the hospital with a Glasgow Coma Score (GCS) of 12 and a carboxyhemoglobin fraction of $30.8 \%$. He was intubated, underwent a left leg escharotomy, and received one treatment with hyperbaric oxygen but was too unstable to tolerate further "dives." In the first $24 \mathrm{~h}$ he received $5.7 \mathrm{~mL} / \mathrm{kg} / \%$ burn resuscitation fluid. He developed acute kidney injury and began CRRT on post-burn day two. Septic shock developed on postburn day five as a result of E. coli, E. faecalis, and S. pneumoniae polymicrobial bacteremia and ventilator-associated pneumonia with methicillin-sensitive Staphylococcus aureus and Haemophilus influenzae, requiring vasopressors, positive-pressure mechanical ventilation, and therapy with vancomycin, piperacillin-tazobactam, and levofloxacin. On post-burn day eight, he developed acalculous cholecystitis and a percutaneous cholecystostomy was placed. The bile yielded E. coli and treatment continued unchanged. Burn excisions were performed on post-operative days 11 and 16 , and tracheostomy and a below-knee amputation of the right leg were performed on post-burn day 18 . 
On post-burn day 21 he developed a surface lesion on his right shoulder; a fungal culture grew $C$. albicans and a blood culture yielded $C$. parapsilosis (both treated with micafungin). Burn dressings were changed to silver nitrate. This initial skin lesion did not contain invasive mold on pathology, but another lesion suspicious for AMI was noted on his right leg on post-burn day 24, with multiple satellite lesions. Multiple biopsies identified polymicrobial AMI, growing Mucor spp., Fusarium spp., A. ustus, Purpureocillium lilacinus, C. albicans, and T. asahii. This infection was treated with intravenous amB and voriconazole. Also on post-burn day 24, a central venous thermoregulation catheter was placed. On post-burn day 25, he underwent extensive debridement of infected soft tissue. Identification of mold on necrotic fascia led to contralateral (left leg) below-knee amputation. On postburn day 29, he was noted to have aggressive recurrence of AMI, now in innumerable locations on both legs and the right arm, so aggressive soft tissue debridement was undertaken again. Topical therapy was switched from silver nitrate to sodium hypochlorite. Despite further debridements on postburn days 32 and 41, the patient continued to deteriorate. A do-not-resuscitate order was written in consultation with the patient's family on post-burn day 52 , and the patient died.

\section{Discussion}

In our three-year experience we had a series of five patients with AMI. Four patients were female and the mean age was 48 y (Table 1). Three patients were African American, one was South Asian, and one was Caucasian. One patient had locally advanced, recurrent breast cancer, and four had large burns (average burn size, 59\% TBSA). Among our burn patients, the average time to develop a mold infection was $25 \mathrm{~d}$ after burn injury. Of interest, four of five patients had polymicrobial mold infection, sometimes with concurrent yeast isolates, which is not reported widely. Included are four isolates of Candida spp., three of Aspergillus spp., two of Fusarium spp., two of T. asahii, two of Mucor spp., and a single isolate of $P$. lilacinus. Purpureocillium lilacinus is known to cause invasive infections [8], although to our knowledge, this is the first reported case of this invasive mold in a burn patient. Four of our patients were treated with intravenous voriconazole, two were treated with intravenous $\mathrm{amB}$, and one was treated with enteral posaconazole. Four patients were treated with topical silver nitrate solution, three were treated with sodium hypochlorite solution, and one was treated with topical amB. Surgical debridement was performed serially on four patients. Three patients were cured of their infections and two patients succumbed, but one patient who had been cured of the AMI died subsequently of another infection; therefore, AMI ultimately portended a $60 \%$ mortality rate in our series. Notably, all of our patients with AMI who died subsequently were burn patients who had more than $50 \%$ TBSA deep burns and developed serious infections and multiple other life-threatening problems prior to their mold infections, including acute kidney injury that required renal replacement therapy, which, in large burns, is associated with $80 \%$ mortality [9]. Moreover, patients with large TBSA burns develop recurrent infections frequently with unusual bacteria, because of injury-related host immunosuppression [10].

In the AMI literature, Aspergillus spp. are the most common cause, however, there has been a recent increase in Fusarium spp. and zygomycetes, such as organisms belonging to the genera Rhizopus and Mucor [7]. A 2008 North American multi-center analysis of 435 positive fungal cultures in burn patients showed the following distribution of

Table 1. Summary of Demographics and Case Details

\begin{tabular}{|c|c|c|c|c|c|}
\hline & Case 1 & Case 2 & Case 3 & Case 4 & Case 5 \\
\hline Age & 70 & 35 & 33 & 65 & 38 \\
\hline Gender & Female & Female & Female & Female & Male \\
\hline $\begin{array}{l}\text { Admission } \\
\text { diagnosis }\end{array}$ & NSTI & $93 \%$ TBSA burn & $68 \%$ TBSA burn & $20 \%$ TBSA burn & $53 \%$ TBSA burn \\
\hline Comorbidities & HTN, Breast Cancer & None & None & COPD, AF, HTN & None \\
\hline $\begin{array}{l}\text { Start of mold } \\
\text { infection }\end{array}$ & HD 12 & PBD 35 & PBD 24 & PBD 18 & PBD 24 \\
\hline \multirow[t]{3}{*}{ Fungal culture } & $\begin{array}{l}\text { Mucor spp. } \\
\text { Candida glabrata }\end{array}$ & $\begin{array}{l}\text { Aspergillus flavus } \\
\text { Aspergillus } \\
\quad \text { calidostus }\end{array}$ & $\begin{array}{l}\text { Fusarium spp. } \\
\text { Candida albicans }\end{array}$ & Aspergillus flavus & $\begin{array}{l}\text { Mucor spp. } \\
\text { Fusarium spp. }\end{array}$ \\
\hline & Candida parapsilosis & $\begin{array}{l}\text { Candida } \\
\quad \text { guilliermondi }\end{array}$ & $\begin{array}{l}\text { Trichosporon } \\
\quad \text { asahii }\end{array}$ & & Aspergillus ustus \\
\hline & & & & & $\begin{array}{l}\text { Purpureocillium } \\
\quad \text { lilacinus } \\
\text { Candida albicans } \\
\text { Trichosporon asahii }\end{array}$ \\
\hline $\begin{array}{l}\text { Systemic } \\
\text { anti-fungal }\end{array}$ & $\begin{array}{c}\text { Amphotericin B, } \\
\text { Posaconazole }\end{array}$ & Voriconazole & Voriconazole & Voriconazole & $\begin{array}{l}\text { Voriconazole, } \\
\text { Amphotericin B }\end{array}$ \\
\hline $\begin{array}{l}\text { Topical } \\
\text { anti-fungal }\end{array}$ & $\begin{array}{l}\text { Sodium hypochlorite } \\
\text { (Dakin), } \\
\text { Amphotericin B }\end{array}$ & Silver nitrate & $\begin{array}{l}\text { Silver nitrate, } \\
\text { sodium } \\
\text { hypochlorite } \\
\text { (Dakin) }\end{array}$ & Silver nitrate & $\begin{array}{l}\text { Silver nitrate, sodium } \\
\text { hypochlorite (Dakin), }\end{array}$ \\
\hline Mold cured? & Yes & Yes & No & Yes & No \\
\hline Disposition & Rehabilitation & Death & Death & Rehabilitation & Death \\
\hline
\end{tabular}

$\mathrm{AF}=$ atrial fibrillation; $\mathrm{COPD}=$ chronic obstructive pulmonary disease; $\mathrm{HD}=$ hospital day; $\mathrm{HTN}=$ hypertension; NSTI = necrotizing soft tissue infection; $\mathrm{PBD}=$ post-burn day; $\mathrm{TBSA}=$ total body surface area. 
organisms: Candida spp., 85\%; non-Candida yeast, 21\%; Aspergillus spp., 14\%; zygomycetes, 9\%; and other fungal organisms, $1.4 \%$ [2]. These same authors found that patients with AMI had an almost 12-fold higher mortality. As a result of their aggressive and morbid nature, these infections need to be treated with both intravenous and topical anti-fungal agents and all visible colonies of mold must be excised, including a margin of adjacent tissue to eradicate residual reservoirs of hyphae. After surgery, systemic and topical antifungal therapy ensures the local control of these infections.

The intravenous treatment of AMI has changed in recent years. In the past, amB and itraconazole were the only available agents. Whereas these were effective against some Aspergillus spp, many non-Aspergillus molds were resistant to these drugs, and the Aspergillus mortality rate was over $80 \%$ in high-risk patients [11]. Currently, voriconazole is the drug of choice for invasive aspergillosis, having been proved more effective than amB in a large, randomized, controlled trial [12]. Voriconazole is a broad-spectrum triazole agent that is fungicidal against all species of Candida, Cryptococcus neoformans, Scedosporium, most species of Aspergillus, and many Fusarium spp [7]. It is also the drug of choice in treating $P$. lilacinus $[13,14]$. However, zygomycetes, including $R h i-$ zopus and Mucor, are resistant to voriconazole [15], and must be treated with amB. Echinocandins may be useful as a second-line agent against Aspergillus, but are not effective against other AMIs [16]. An echinocandin may sometimes used in combination with voriconazole [17], but there are no controlled clinical trials showing that combination therapy is superior. Whereas culture and susceptibility testing should guide antimicrobial therapy, voriconazole should be the firstline drug for Aspergillus infections and amB should be firstline therapy for zygomycetes. Combination therapy with posaconazole has been reported to be of benefit to treat zygomycetes, particularly as salvage therapy [18-21]. Whereas voriconazole may be an acceptable empiric agent, as it will be effective against most AMIs, combination therapy with both voriconazole and amB may be justifiable sometimes as empiric therapy, with de-escalation after microbiologic results become available [4]. It is unknown for the most part whether combination therapy is efficacious and safe [22]. Clinical trials are sorely needed.

Topical anti-fungal agents include nystatin, silvercontaining compounds, amB, and sodium hypochlorite solution [5]. All of these agents are, to some degree, cytotoxic to normal tissue as well. As a result, their use may compromise autografts in the area of application. When using topical nystatin, a relatively high concentration is needed $(>6,000,000 \mathrm{U} / \mathrm{g})$ to treat AMI, whereas lower concentrations may be sufficient for Candida spp. [23,24]. Silver-containing compounds, including silver nitrate and silver sulfadiazine have been welldemonstrated to have efficacy against nearly all species of Candida, Aspergillus, Fusarium, and zygomycetes [25,26]. Conventional amB is occasionally used topically, including in pulse lavage devices, although topical formulations of these drugs may become more widely available and effective in the form of amB-releasing nanoparticles [4,27,28]. Most experience with conventional topical amB is reported for rhinosinusitis and rhinocerebral infections [29,30], although effectiveness is dubious because amB has poor solubility in aqueous solution. Trans-dermal absorption of amB may also be enhanced by encapsulation in positively charged (stear- ylamine) hydrogenated soya phosphatidylcholine/cholesterol/ charged lipid liposomes [31]. Topical use of amB lipid formulations available clinically has been reported only sporadically, to treat keratitis and cutaneous leishmaniasis [32], but never for AMI. Topical sodium hypochlorite solution is used less commonly in burn centers, but it does have anti-fungal properties, and has been used successfully to treat AMI in multiple case series, particularly in the military medical literature $[4,33]$. There are no data to suggest strongly the superiority of any particular topical anti-fungal agent at this time, however, amB is plausibly a particularly effective agent for treating zygomycetes. The use of topical anti-fungal agents is not universal, but, given the high risk of mortality in these infections, a risk-benefit analysis of benefit as an adjunct to surgery and systemic anti-fungal therapy may clarify the potential for use in proximity to recent split-thickness skin grafts.

\section{Conclusion}

Preventing AMI is difficult because Aspergillus, zygomycetes, and other mold species are ubiquitous organisms, most commonly present in decaying organic matter. Prohibition of flowers and other organic fomites is common practice in all hospitals caring for at-risk patients. Thus, early burn excision as soon as surgery can be tolerated is a means to remove decaying organic material from the body. Additionally, application of topical antimicrobial agents to open wounds, including nystatin or silver-containing compounds with antifungal properties, is possibly of utility. Rapid removal of foreign bodies no longer needed, including endotracheal tubes, Foley catheters, and arterial and central venous catheters is also important. Rational circumscribed use of broad spectrum antibiotics will promote host bacterial ecology and reduce opportunistic infection. Other basic factors to support immune competence include tight glycemic control, early enteral nutrition, and restrictive transfusion of blood products. However, because of the ubiquitous nature of mold, infections can occur even in the most fastidious clinical environment. At such times, rapid diagnosis by obtaining biopsy, culture, and sensitivities; prompt initiation of empiric systemic and topical anti-fungal treatment, and aggressive surgical control are essential to optimal outcomes.

\section{Author Disclosure Statement}

The authors have no conflicts of interest or disclosures. This work has not been previously presented and is not in consideration for publication at any other journals

\section{References}

1. Horvath EE, Murray CK, Vaughan GM, et al. Fungal wound infection (not colonization) is independently associated with mortality in burn patients. Ann Surg 2007;245: 978-985.

2. Ballard J, Edelman L, Saffle J, et al. Positive fungal cultures in burn patients: A multicenter review. J Burn Care Res 2008;29:213-221.

3. Petrikkos G, Skiada A, Lortholary O, et al. Epidemiology and clinical manifestations of mucormycosis. Clin Infect Dis 2012;54(Suppl 1):S23-S34.

4. Warkentien T, Rodriguez C, Lloyd B, et al. Invasive mold infections following combat-related injuries. Clin Infect Dis 2012;55:1441-1449. 
5. Struck MF, Gille J. Fungal infections in burns: A comprehensive review. Ann Burns Fire Disasters 2013;26:147153.

6. Capoor MR, Sarabahi S, Tiwari VK, Narayanan RP. Fungal infections in burns: Diagnosis and management. Indian $\mathrm{J}$ Plast Surg 2010;43:0970-0358.

7. Kauffman CA. Fungal infections. Proc Am Thorac Soc 2006;3:35-40.

8. Khan Z, Ahmad S, Al-Ghimlas F, et al. Purpureocillium lilacinum as a cause of cavitary pulmonary disease: A new clinical presentation and observations on atypical morphologic characteristics of the isolate. J Clin Microbiol 2012;50:1800-1804.

9. Ibrahim AE, Sarhane KA, Fagan SP, Goverman J. Renal dysfunction in burns: A review. Ann Burns Fire Disasters 2013;26:16-25.

10. Pirnay JP, De Vos D, Cochez C, et al. Molecular epidemiology of Pseudomonas aeruginosa colonization in a burn unit: Persistence of a multidrug-resistant clone and a silver sulfadiazineresistant clone. J Clin Microbiol 2003;41:1192-1202.

11. Lin SJ, Schranz J, Teutsch SM. Aspergillosis case-fatality rate: Systematic review of the literature. Clin Infect Dis 2001;32:358-366.

12. Herbrecht R, Denning DW, Patterson TF, et al. Voriconazole versus amphotericin B for primary therapy of invasive aspergillosis. N Engl J Med 2002;347:408-415.

13. Pastor FJ, Guarro J. Clinical manifestations, treatment and outcome of Paecilomyces lilacinus infections. Clin Microbiol Infect 2006;12:948-960.

14. Rodriguez MM, Pastor FJ, Serena C, Guarro J. Efficacy of voriconazole in a murine model of invasive paecilomycosis. Int J Antimicrob Agents 2010;35:362-365.

15. Johnson LB, Kauffman CA. Voriconazole: A new triazole antifungal agent. Clin Infect Dis 2003;36:630-637.

16. Maertens J, Raad I, Petrikkos G, et al. Efficacy and safety of caspofungin for treatment of invasive aspergillosis in patients refractory to or intolerant of conventional antifungal therapy. Clin Infect Dis 2004;39:1563-1571.

17. Marr KA, Boeckh M, Carter RA, et al. Combination antifungal therapy for invasive aspergillosis. Clin Infect Dis 2004;39:797-802.

18. Enoch DA, Aliyu SH, Sule O, et al. Posaconazole for the treatment of mucormycosis. Int J Antimicrob Agents 2011;38: 465-473.

19. Rickerts V, Atta J, Herrmann S, et al. Successful treatment of disseminated mucormycosis with a combination of liposomal amphotericin B and posaconazole in a patient with acute myeloid leukemia. Mycoses 2006;1:27-30.

20. Sun QN, Najvar LK, Bocanegra R, et al. In vivo activity of posaconazole against Mucor spp. in an immunosuppressedmouse model. Antimicrob Agents Chemother 2002;46:2310 2312.

21. Vehreschild JJ, Birtel A, Vehreschild MJ, et al. Mucormycosis treated with posaconazole: Review of 96 case reports. Crit Rev Microbiol 2013;39:310-324.

22. Spellberg B, Ibrahim A, Roilides E, et al. Combination therapy for mucormycosis: Why, what, and how? Clin Infect Dis 2012;54(Suppl 1):S73-S78.

23. Barret JP, Ramzy PI, Heggers JP, et al. Topical nystatin powder in severe burns: A new treatment for angioinvasive fungal infections refractory to other topical and systemic agents. Burns 1999;25:505-508.

24. Church D, Elsayed S, Reid O, et al. Burn wound infections. Clin Microbiol Rev 2006;19:403-434.
25. Xu Y, Pang GR, Gao CW, et al. [Comparison of the activities of silver nitrate with those of three antifungal agents against ocular pathogenic fungi in vitro.] Zhonghua Yan Ke Za Zhi 2009;45:730-735.

26. Wright JB, Lam K, Hansen D, Burrell RE. Efficacy of topical silver against fungal burn wound pathogens. Am J Infect Control 1999;27:344-350.

27. Resch TR, Main S, Price LA, Milner SM. A fungal burn infection. Eplasty 2014;14:ic5.

28. Sanchez DA, Schairer D, Tuckman-Vernon C, et al. Amphotericin B releasing nanoparticle topical treatment of Candida spp. in the setting of a burn wound. Nanomedicine 2014;10:269-277.

29. Wang T, Su J, Feng Y. The effectiveness topical amphotericin B in the management of chronic rhinosinusitis: A metaanalysis. Eur Arch Otorhinolaryngol 2015;272:1923-1929.

30. Saedi B, Sadeghi M, Seilani P. Endoscopic management of rhinocerebral mucormycosis with topical and intravenous amphotericin B. J Laryngol Otol 2011;125:807-810.

31. Manosroi A, Kongkaneramit L, Manosroi J. Stability and transdermal absorption of topical amphotericin B liposome formulations. Int J Pharm 2004;270:279-286.

32. Layegh P, Rajabi O, Jafari MR, et al. Efficacy of topical liposomal amphotericin B versus intralesional meglumine antimoniate (glucantime) in the treatment of cutaneous leishmaniasis. J Parasitol Res 2011;2011:656523.

33. Lewandowski L, Purcell R, Fleming M, Gordon WT. The use of dilute Dakin's solution for the treatment of angioinvasive fungal infection in the combat wounded: A case series. Mil Med 2013;178:12-00322.

Address correspondence to: Dr. Jason C. Gardenier William Randolph Hearst Burn Center New York-Presbyterian Hospital

Weill Cornell Medicine 525 East 68th Street Box 137

New York, NY 10065

E-mail: jcg9009@med.cornell.edu

$\begin{aligned} & \text { Abbreviations Used } \\ \text { amB } & =\text { amphotericin B } \\ \mathrm{AMI} & =\text { angioinvasive mold infections } \\ \mathrm{CRRT} & =\text { continuous renal replacement therapy } \\ \mathrm{GMS} & =\text { Golcott-Gomeri methenamine silver } \\ \mathrm{ICU} & =\text { intentive care unit } \\ \mathrm{KOH} & =\text { potossium chloride } \\ \mathrm{NSTI} & =\text { necrotizing soft tissue infection } \\ \mathrm{PAS} & =\text { periodic acid-Schiff } \\ \mathrm{TBSA} & =\text { total body surface area }\end{aligned}$

Cite this article as: Gardenier JC, Chopra VK, Filicori F, Murphy J, Greenway A, Gallagher JJ, Bessey PQ, Houng A, Eachempati SR, Barie PS (2016) Angioinvasive mold in the surgical and burn intensive care unit: A case series and review of the literature. Surgical Infections Case Reports 1:1, 72-78, DOI: 10.1089/crsi.2016.0016. 